November 2, 2018

\title{
The distribution of the maximum of a first order moving average: the continuous case http://arxiv.org/abs/0802.0523
}

by

\author{
Christopher S. Withers 1 \\ Applied Mathematics Group \\ Industrial Research Limited \\ Lower Hutt, NEW ZEALAND \\ Saralees Nadarajah \\ School of Mathematics \\ University of Manchester \\ Manchester M60 1QD, UK
}

\begin{abstract}
We give the distribution of $M_{n}$, the maximum of a sequence of $n$ observations from a moving average of order 1 . Solutions are first given in terms of repeated integrals and then for the case where the underlying independent random variables have an absolutely continuous density. When the correlation is positive,$$
P\left(M_{n} \leq x\right)=\sum_{j=1}^{\infty} \beta_{j x} \nu_{j x}^{n} \approx B_{x} \nu_{1 x}^{n}
$$

where $\left\{\nu_{j x}\right\}$ are the eigenvalues (singular values) of a Fredholm kernel and $\nu_{1 x}$ is the eigenvalue of maximum magnitude. A similar result is given when the correlation is negative. The result is analogous to large deviations expansions for estimates, since the maximum need not be standardized to have a limit.

For the continuous case the integral equations for the left and right eigenfunctions are converted to first order linear differential equations. The eigenvalues satisfy an equation of the form

$$
\sum_{i=1}^{\infty} w_{i}\left(\lambda-\theta_{i}\right)^{-1}=\lambda-\theta_{0}
$$
\end{abstract}

for certain known weights $\left\{w_{i}\right\}$ and singular values $\left\{\theta_{i}\right\}$ of a given matrix. This can be solved by truncating the sum to an increasing number of terms.

\section{Introduction and Summary}

Little is available in the literature on the behaviour of extremes of correlated sequences apart from some special cases involving Gaussian processes and some weak convergence results. For example

\footnotetext{
${ }^{1}$ Work begun while visiting the Statistics Dept, UNC, Chapel Hill.
} 
Leadbetter et al. (1983) p59 give a convergence in distribution for the scaled maximum of a stationary sequence, and Resnick (1987) p239 gives a similar result for moving averages.

This paper gives a powerful new method for giving the exact distribution of extremes of $n$ correlated observations as weighted sums of $n$th powers of associated eigenvalues. The method is illustrated here for a moving average of order 1 .

Let $\left\{e_{i}\right\}$ be independent and identically distributed random variables from some distribution $F$ on $R$. Consider the moving average of order 1 ,

$$
X_{i}=e_{i}+\rho e_{i-1}
$$

where $\rho \neq 0$. In Section 2 we give expressions for the distribution of the maximum

$$
M_{n}=\max _{i=1}^{n} X_{i}
$$

in terms of repeated integrals. This is obtained via the recurrence relationship

$$
\begin{aligned}
G_{n}(y) & =I(\rho<0) G_{n-1}(\infty) F(y)+\mathcal{K} G_{n-1}(y) \\
\text { where } G_{n}(y) & =P\left(M_{n} \leq x, e_{n} \leq y\right),
\end{aligned}
$$

$I(A)=1$ or 0 for $A$ true or false, and $\mathcal{K}$ is an integral operator depending on $x$. (Dependence on $x$ is suppressed.) For this to work at $n=1$ we define

$$
M_{0}=-\infty \text { so that } G_{0}(y)=F(y) .
$$

In Section 3 we consider the case when $F$ is absolutely continuous with density $f(x)$ with respect to Lebesque measure. In this case we show that corresponding to $\mathcal{K}$ is a Fredholm kernel $K(y, z)$. We give a solution in terms of its eigenvalues and eigenfunctions. This leads easily to the asymptotic results stated in the abstract.

Our expansions for $P\left(M_{n} \leq x\right)$ for fixed $x$ are large deviation results. If $x$ is replaced by $x_{n}$ such that $P\left(M_{n} \leq x_{n}\right)$ tends to the GEV distribution, then the expansion still holds, but not the asymptotic expansion in terms of a single eigenvalue, since this may approach 1 as $n \rightarrow \infty$.

Set $\int r=\int r(y) d y$.

\section{Solutions using repeated integrals.}

For $n \geq 1, G_{n}$ of (1.2) satisfies

$$
\begin{aligned}
G_{n}(y) & =P\left(M_{n-1} \leq x, e_{n}+\rho e_{n-1} \leq x, e_{n} \leq y\right) \\
& =P\left(M_{n-1} \leq x, e_{n-1} \leq\left(x-e_{n}\right) / \rho, e_{n} \leq y\right) \text { if } \rho>0 \\
& =\int^{y} G_{n-1}((x-w) / \rho) d F(w) \\
& =P\left(M_{n-1} \leq x, e_{n-1} \geq\left(x-e_{n}\right) / \rho, e_{n} \leq y\right) \text { if } \rho<0 \\
& =\int^{y}\left[G_{n-1}(\infty)-G_{n-1}((x-w) / \rho)\right] d F(w)=G_{n-1}(\infty) F(y)-\int^{y} G_{n-1}((x-w) / \rho) d F(w) .
\end{aligned}
$$

(Thanks to the referee for noting a slip in the last line.) That is, for $n \geq 1$, (1.1) holds with

$$
\mathcal{K} r(y)=\operatorname{sign}(\rho) \int^{y} r((x-w) / \rho) d F(w) .
$$


Our goal is to determine

$$
u_{n}=P\left(M_{n} \leq x\right)=G_{n}(\infty) .
$$

In this section we give $u_{n}$ in terms of

$$
v_{n}=\left[\mathcal{K}^{n} F(y)\right]_{y=\infty} .
$$

For example

$$
v_{1}=-\int F(z) d F(x-\rho z)=-I(\rho<0)+\int F(x-\rho z) d F(z) .
$$

The behaviour of $u_{n}$ falls into two cases.

The case $\rho>0$. For $n \geq 1$,

$$
u_{n}=v_{n}
$$

since

$$
G_{n}(y)=\mathcal{K}^{n} F(y)
$$

The marginal distribution of $X_{1}$ is $u_{1}=v_{1}$ given by (2.3).

The case $\rho<0$. By (1.1), for $n \geq 0$,

$$
\begin{aligned}
G_{n+1}(y) & =u_{n} F(y)+\mathcal{K} G_{n}(y) \\
& =a_{n}(y) \otimes u_{n}+a_{n+1}(y) \text { where } a_{i}(y)=\mathcal{K}^{i} F(y), a_{n} \otimes b_{n}=\sum_{j=0}^{n} a_{j} b_{n-j} .
\end{aligned}
$$

Putting $y=\infty$ gives the recurrence equation for $u_{n}$ :

$$
u_{0}=1, u_{n+1}=v_{n+1}+\sum_{i=0}^{n} v_{i} u_{n-i}, n \geq 0 .
$$

The marginal distribution of $X_{1}$ is $u_{1}=1+v_{1}$ of (2.3).

An explicit solution for $u_{n}$ when $\rho<0$.

Define the generating functions

$$
U(t)=\sum_{n=0}^{\infty} u_{n} t^{n}, V(t)=\sum_{n=0}^{\infty} v_{n} t^{n},
$$

Multiplying (2.6) by $t^{n}$ and summing from $n=0$ gives $(U(t)-V(t)) / t=U(t) V(t)$, so that

$$
\begin{aligned}
U(t) & =(1-W(t))^{-1} V(t) \text { where } W(t)=t V(t)=\sum_{n=1}^{\infty} w_{n} t^{n}, w_{n}=v_{n-1}, \\
t U(t) & =(1-W(t))^{-1} W(t)=(1-W(t))^{-1}-1=\sum_{j=1}^{\infty} W(t)^{j} .
\end{aligned}
$$

By definition, for $j=0,1, \cdots$

$$
W(t)^{j}=\sum_{n=j}^{\infty} \hat{B}_{n j}(w) t^{n}
$$


where $\hat{B}_{n j}(w)$ is the partial ordinary Bell polynomial in $w=\left(w_{1}, w_{2}, \cdots\right)$ tabled on p309 of Comtet (1974). For example

$$
\hat{B}_{n 0}(w)=\delta_{n 0}, \hat{B}_{n 1}(w)=w_{n}, \hat{B}_{n 1}(w)=w_{1}^{n} .
$$

So

$$
t U(t)=(1-W(t))^{-1}-1=\sum_{n=1}^{\infty} \hat{B}_{n}(w) t^{n}
$$

where

$$
\hat{B}_{n}(w)=\sum_{j=0}^{n} \hat{B}_{n j}(w)
$$

is the complete ordinary Bell polynomial. For example $\hat{B}_{0}(w)=1$. Taking the coefficient of $t^{n}$ gives the explicit solution

$$
u_{n-1}=\hat{B}_{n}(w), n \geq 1 \text {. }
$$

For example

$$
\begin{aligned}
& u_{0}=\hat{B}_{1}(w)=\hat{B}_{11}(w)=w_{1}=v_{0}=1, \\
& u_{1}=\hat{B}_{2}(w)=\hat{B}_{21}(w)+\hat{B}_{22}(w)=w_{2}+w_{1}^{2}=v_{1}+1 .
\end{aligned}
$$

Similarly from Comtet's table we can immediately read off $u_{n}, 1 \leq n \leq 9$ :

$$
\begin{aligned}
u_{0}= & 1, u_{1}=v_{1}+1 \\
u_{2}= & v_{2}+2 v_{1}+1 \\
u_{3}= & v_{3}+\left(2 v_{2}+v_{1}^{2}\right)+3 v_{1}+1 \\
u_{4}= & v_{4}+\left(2 v_{3}+2 v_{1} v_{2}\right)+\left(3 v_{2}+3 v_{1}^{2}\right)+4 v_{1}+1, \\
u_{5}= & v_{5}+\left(2 v_{4}+2 v_{1} v_{3}+v_{2}^{2}\right)+\left(3 v_{3}+6 v_{1} v_{2}+v_{1}^{3}\right)+\left(4 v_{2}+6 v_{1}^{2}\right)+5 v_{1}+1, \\
u_{6}= & v_{6}+\left(2 v_{5}+2 v_{1} v_{4}+2 v_{2} v_{3}\right)+\left(3 v_{4}+6 v_{1} v_{3}+3 v_{2}^{2}+3 v_{1}^{2} v_{2}\right)+\left(4 v_{3}+12 v_{1} v_{2}+4 v_{1}^{3}\right) \\
& +\left(5 v_{2}+10 v_{1}^{2}\right)+6 v_{1}+1 \\
u_{7}= & v_{7}+\left(2 v_{6}+2 v_{1} v_{5}+2 v_{2} v_{4}+v_{3}^{2}\right)+\left(3 v_{5}+6 v_{1} v_{4}+6 v_{2} v_{3}+3 v_{1}^{2} v_{3}+3 v_{1} v_{2}^{2}\right) \\
& +\left(4 v_{4}+12 v_{1} v_{3}+6 v_{2}^{2}+12 v_{1}^{2} v_{2}+v_{1}^{4}\right)+\left(5 v_{3}+20 v_{1} v_{2}+10 v_{1}^{3}\right)+\left(6 v_{2}+15 v_{1}^{2}\right)+7 v_{1}+1, \\
u_{8}= & v_{8}+\left(2 v_{7}+2 v_{1} v_{6}+2 v_{2} v_{5}+2 v_{3} v_{4}\right)+\left(3 v_{6}+6 v_{1} v_{5}+6 v_{2} v_{4}+3 v_{3}^{2}+3 v_{1}^{2} v_{4}+6 v_{1} v_{2} v_{3}+v_{2}^{3}\right) \\
& +\left(4 v_{5}+12 v_{1} v_{4}+12 v_{2} v_{3}+12 v_{1}^{2} v_{3}+12 v_{1} v_{2}^{2}+4 v_{1}^{3} v_{2}\right)+\left(5 v_{4}+20 v_{1} v_{3}+10 v_{2}^{2}+30 v_{1}^{2} v_{2}+5 v_{1}^{4}\right) \\
& +\left(6 v_{3}+30 v_{1} v_{2}+20 v_{1}^{3}\right)+\left(7 v_{2}+21 v_{1}^{2}\right)+8 v_{1}+1, \\
u_{9}= & v_{9}+\left(2 v_{8}+2 v_{1} v_{7}+2 v_{2} v_{6}+2 v_{3} v_{5}+v_{4}^{2}\right)+\left(3 v_{7}+6 v_{1} v_{6}+6 v_{2} v_{5}+6 v_{3} v_{4}+3 v_{1}^{2} v_{5}+6 v_{1} v_{2} v_{4}\right. \\
& \left.+3 v_{1} v_{3}^{2}+3 v_{2}^{2} v_{3}\right)+\left(4 v_{6}+12 v_{1} v_{5}+12 v_{2} v_{4}+6 v_{3}^{2}+12 v_{1}^{2} v_{4}+24 v_{1} v_{2} v_{3}+4 v_{2}^{3}+4 v_{1}^{3} v_{3}\right. \\
& \left.+6 v_{1}^{2} v_{2}^{2}\right)+\left(5 v_{5}+20 v_{1} v_{4}+20 v_{2} v_{3}+30 v_{1}^{2} v_{3}+30 v_{1} v_{2}^{2}+20 v_{1}^{3} v_{2}+v_{1}^{5}\right)+\left(6 v_{4}+30 v_{1} v_{3}+15 v_{2}^{2}\right. \\
& \left.+60 v_{1}^{2} v_{2}+15 v_{1}^{4}\right)+\left(7 v_{3}+42 v_{1} v_{2}+35 v_{1}^{3}\right)+\left(8 v_{2}+28 v_{1}^{2}\right)+9 v_{1}+1 .
\end{aligned}
$$

More generally any $u_{n}$ can be obtained from (2.7) using the recurrence relation

$$
b_{n}=w_{n} \otimes b_{n}, n \geq 1, \text { where } w_{0}=0, b_{n}=\hat{B}_{n}(w) .
$$

For example since $b_{0}=1$, this gives

$$
b_{1}=w_{1}, b_{2}=w_{1}^{2}+w_{2}, b_{3}=w_{1}^{3}+2 w_{1} w_{2}+w_{3} .
$$

The recurrence relation (2.8) for the complete ordinary Bell polynomials follows by taking the coefficient of $t^{n}$ in $(1-w)^{-1}-1=w(1-w)^{-1}$ where $w=W(t)$, and appears to be new. 


\section{The absolutely continuous case.}

Our solutions (2.4), (2.6), (3.8) do not tell us how $u_{n}$ behaves for large $n$. Also they require repeated integration. Here we give solutions that overcome these problems, using Fredholm integral theory given in Appendix A. Write (2.1) in the form

$$
\mathcal{K} r(y)=\int K(y, z) r(z) d z \text { where } K(y, z)=\rho I(x \leq y+\rho z) f(x-\rho z) .
$$

Since

$$
\begin{aligned}
\|\mathcal{K}\|_{2}^{2}=\iint K(y, z) K(z, y) d y d z & =\rho^{2} \iint I(x<y+\rho z) I(x<z+\rho y) f(x-\rho z) f(x-\rho y) d y d z \\
& <\rho^{2} \iint f(x-\rho z) f(x-\rho y) d y d z=1
\end{aligned}
$$

$K(y, z)$ is said to be a Fredholm kernel w.r.t. Lebesgue measure, allowing the Fredholm theory of the Appendix to be applied, in particular the functional forms of the Jordan form and singular value decomposition. If say, $0<\rho<1$, then one can show that

$$
\|\mathcal{K}\|_{2}^{2}=\int F\left(x_{t}\right) d F(t) \uparrow 1 \text { as } x \uparrow \infty \text { where } x_{t}=\min (x-\rho t,(x-t) / \rho) .
$$

Let $\left\{\lambda_{j}, r_{j}, l_{j}: j \geq 1\right\}$ be its eigenvalues (singular values) and associated right and left eigenfunctions ordered so that $\left|\lambda_{j}\right| \leq\left|\lambda_{j+1}\right|$. By Appendix A these satisfy

$$
\lambda_{j} \mathcal{K} r_{j}=r_{j}, \lambda_{j} l_{j} \mathcal{K}=l_{j}, \int r_{j} l_{k}=\delta_{j k}
$$

where $\delta_{j k}$ is the Kronecker function and we write $\int a(y) b(y) d y=\int a b$. So $\left\{r_{j}(y), l_{k}(y)\right\}$ are biorthogonal functions with respect to Lebesgue measure. Set

$$
\nu_{j}=1 / \lambda_{j}
$$

By (3.2) and (A.6),

$$
1>\|\mathcal{K}\|_{2}^{2}=\sum_{j=1}^{\infty} \nu_{j}^{2}
$$

where $\nu_{j}$ are the singular values, or if the Jordan form is diagonal, the eigenvalues. (We shall use these terms interchangeably.) So $\left|\nu_{j}\right|<1$ and $1+\nu_{j}>0$.

Consider the case where the Jordan form is diagonal. Suppose that the eigenvalue $\lambda_{1}$ of smallest magnitude has multiplicity $M$ (typically 1 ). Set

$$
\beta_{j}=r_{j}(\infty) \int F l_{j}, B=\sum_{j=1}^{M} \beta_{j} .
$$

Then by (A.8) for $n \geq 1$,

$$
v_{n}=\sum_{j=1}^{\infty} \beta_{j} \nu_{j}^{n}=B \nu_{1}^{n}\left(1+\epsilon_{n}\right)
$$


where $\epsilon_{n} \rightarrow 0$ exponentially as $n \rightarrow \infty$. (In fact by (A.8) $1=v_{0}=\sum_{j=1}^{\infty} \beta_{j}$ if this converges.) So for $n \geq 1$, by (2.4)

$$
\text { for } \rho>0, u_{n}=\sum_{j=1}^{\infty} \beta_{j} \nu_{j}^{n} .
$$

$\nu_{1}$ is given by (A.10) with $\mu$ Lebesgue measure. (When $\rho=0$ then (3.6) holds with $\beta_{j}=\delta_{j 1}, \nu_{1}=$ $F(x)$. So we expect that $\nu_{1} \rightarrow F(x)$ as $\rho \downarrow 0$.)

Now suppose that $\rho<0$.

By (3.5), for $\max _{j=1}^{\infty}\left|\nu_{j} t\right|<1, V(t)=1+\sum_{j=1}^{\infty} \beta_{j} \nu_{j} t /\left(1-\nu_{j} t\right)$. So

$$
\begin{aligned}
1-t V(t) & =1-t-\sum_{j=1}^{\infty} \beta_{j} \nu_{j} t^{2} /\left(1-\nu_{j} t\right)=N(t) / D(t) \\
\text { where } D(t) & =\Pi_{j=1}^{\infty}\left(1-\nu_{j} t\right), N(t)=\Pi_{j=1}^{\infty}\left(1-w_{j} t\right) \text { say. }
\end{aligned}
$$

$D(t)$ is the Fredholm determinant of $K(x, y)$. (Now $w_{j}$ takes on a different meaning than in Section 2.) So by the partial fraction expansion, assuming that $\left\{w_{j}\right\}$ are all different,

$$
\begin{aligned}
N(t)^{-1} & =\sum_{j=1}^{\infty} c_{j}^{-1}\left(1-w_{j} t\right)^{-1} \text { where } c_{j}=\Pi_{k \neq j}\left(1-w_{k} / w_{j}\right), \\
& =\sum_{n=0}^{\infty} N_{n} t^{n} \text { where } N_{n}=\sum_{j=1}^{\infty} c_{j}^{-1} w_{j}^{n} .
\end{aligned}
$$

Also by Fredholm's first theorem - see for example, p47 of Pogorzelski (1966),

$$
D(t)=1+\sum_{n=1}^{\infty} D_{n}(-t)^{n} / n !, D_{n}=\int \cdots \int N\left(\begin{array}{l}
s_{1} \cdots s_{n} \\
s_{1} \cdots s_{n}
\end{array}\right) d s_{1} \cdots d s_{n}
$$

where $N\left(\begin{array}{l}s_{1} \cdots s_{n} \\ s_{1} \cdots s_{n}\end{array}\right)=\operatorname{det}\left(N\left(s_{j}, s_{k}\right), 1 \leq j, k \leq n\right)$. Alternatively, a simple expansion gives

$$
D_{n} / n !=\sum_{1 \leq j_{1}<\cdots<j_{n}} \nu_{j_{1}} \cdots \nu_{j_{n}}=\left[1^{n}\right]
$$

the augmented symmetric function, in the notation of Table 10 of Stuart and Ord (1987). This table gives $\left[1^{n}\right]$ in terms of the power sums $(r)=\sum_{j=1}^{\infty} \nu_{j}^{r}$. For example $\left[1^{3}\right]=2(3)-(2)(1)+(1)^{3}$. In our case

$$
(r)=\int K_{r}(x, x) d x=\sum_{j=1}^{\infty} \nu_{j}^{r}
$$

where by (A.4)

$$
K_{r}(x, y)=\mathcal{K}^{r-1} K(x, y)=\sum_{j=1}^{\infty} \nu_{j}^{r} r_{j}(x) l_{j}(y)
$$

So $\left[1^{n}\right]$ has the form

$$
\left[1^{n}\right]=\sum_{k=1}^{n} \sum_{n_{1}+\cdots+n_{k}=n} A\left(n_{1} \cdots n_{k}\right)\left(n_{1}\right) \cdots\left(n_{k}\right)
$$


However this does not give its behaviour for large $n$. At any rate, we have

$$
1+t U(t)=(1-W(t))^{-1}=(1-t V(t))^{-1}=D(t) / N(t)
$$

so that

$$
u_{n-1}=N_{n} \otimes D_{n}(-1)^{n} / n !
$$

where $N_{n}$ is given by (3.7). This solution will be useful for large $n$ if $D_{n}$ has an expansion of the form (3.5). However to date we have not been able to show this directly. One can show that $D_{n}=(-1)^{n} B_{n}(d)$ where $B_{n}(d)$ is the complete exponential Bell polynomial, $d_{r}=-(r-1) ! w(n)$, and $w(n)=\sum_{j=1}^{\infty} w_{j}^{n}=(n)$ for $w$. We conjecture that if $d_{n}=\sum_{j=1}^{\infty} a_{j} w_{j}^{n}$ where $\left|w_{j}\right|$ is strictly decreasing and $\left|a_{j}\right|>1$, then

$$
B_{n}(d) \approx d_{n} \approx a_{1} w_{1}^{n} \text { as } n \rightarrow \infty
$$

An alternative approach is to try a solution for $u_{n}$ of the form (3.5), say

$$
u_{n}=\sum_{j=1}^{\infty} \gamma_{j} \delta_{j}^{n}
$$

where $\delta_{j}$ decrease in magnitude. Assuming that $\left\{\delta_{j}, \nu_{j}\right\}$ are all distinct, substitution into the recurrence relation (2.6) gives us the following elegant relations. $\left\{\delta_{j}\right\}$ are the roots of

$$
\sum_{k=1}^{\infty} \beta_{k} /\left(\delta-\nu_{k}\right)=1
$$

and $\beta_{k}$ is given by (3.4). Having found $\left\{\delta_{j}\right\},\left\{\gamma_{j}\right\}$ are the roots of

$$
\sum_{j=1}^{\infty} \gamma_{j} /\left(\delta_{j}-\nu_{k}\right) \equiv 1
$$

The last equation can be written

$$
A \gamma=\mathbf{1} \text { where } A=\left(A_{k j}: k, j \geq 1\right), A_{k j}=1 /\left(\delta_{j}-\nu_{k}\right) .
$$

So a formal solution is

$$
\gamma=A^{-1} \mathbf{1}
$$

Numerical solutions can be found by truncating the infinite matrix $A$ and infinite vectors $\mathbf{1}, \gamma$ to $N \times N$ matrix and $N$-vectors, then increasing $N$ until the desired precision is reached.

\section{Behaviour for large $n$ and $x$ independent of $n$.}

For $\rho>0$, (3.6) implies

$$
u_{n} \approx B \nu_{1}^{n} \text { and } \nu_{1}>0
$$

where $B$ is given by (3.4). Also $\nu_{1}$ is given by (A.10) with $\mu$ Lebesgue measure.

Now suppose that $\rho<0$. By (3.9),

$$
u_{n}=\gamma_{1} \delta_{1}^{n}\left(1+\epsilon_{n}^{\prime}\right)
$$


where $\epsilon_{n}^{\prime} \rightarrow 0$ exponentially as $n \rightarrow \infty$ and $\delta_{1}$ has the largest magnitude among $\left\{\delta_{n}\right\}$. (The case where multiple $\delta_{n}$ exist of magnitude $\left|\delta_{1}\right|$ requires an obvious adaptation.)

\section{Integral and differential equations for the eigenfunctions and resolvent.}

The right eigenfunctions satisfy $\nu_{j} r_{j}=\mathcal{K} r_{j}$, that is

$$
\nu_{j} r_{j}(y)=\mathcal{K} r_{j}(y)=\operatorname{sign}(\rho) \int^{y} r_{j}((x-w) / \rho) d F(w)
$$

For example

$$
\nu_{j} r_{j}(\infty)=\rho \int r_{j}(z) f(x-\rho z) d z .
$$

Differentiating gives the non-standard linear first order differential equation

$$
\nu_{j} \dot{r}_{j}(y)=\operatorname{sign}(\rho) f(y) r_{j}((x-y) / \rho), r_{j}(-\infty)=0 .
$$

Similarly the left eigenfunctions satisfy

$\nu_{j} l_{j}=l_{j} \mathcal{K}$, that is

$$
\nu_{j} l_{j}(z)=\int l_{j}(y) K(y, z) d y=\rho f(x-\rho z) \int_{x-\rho z} l_{j}(y) d y .
$$

So

$$
l_{j}(-\infty)=0 \text { if } \rho>0, l_{j}(\infty)=0 \text { if } \rho<0
$$

and by differentiating,

$$
\nu_{j}(d / d z)\left[l_{j}(z) / f(x-\rho z)\right]=\rho^{2} l_{j}(x-\rho z) .
$$

The resolvent satisfies

$$
[K(y, z, \lambda)-K(y, z)] / \lambda=\mathcal{K} K(y, z, \lambda)=K(y, z, \lambda) \mathcal{K} .
$$

So

$$
\begin{aligned}
K(-\infty, z, \lambda) & =0, \\
K(y, \infty, \lambda) & =0 \text { if } \rho<0, \\
K(y,-\infty, \lambda) & =0 \text { if } \rho>0
\end{aligned}
$$

and by differentiation the resolvent satisfies the first order partial differential equations

$$
\begin{aligned}
(\partial / \partial y) \operatorname{LHS}(3.19) & =\operatorname{sign}(\rho) f(y) K((x-y) / \rho, z, \lambda), \\
(\partial / \partial z)[\operatorname{LHS}(3.19) / f(x-\rho z)] & =\rho^{2} K(y, x-\rho z, \lambda) .
\end{aligned}
$$

These may involve the Dirac function $\delta(x)$ since with $x_{z}=x-\rho z$,

$$
\begin{aligned}
& (\partial / \partial y) K(y, z)=\rho f\left(x_{z}\right) \delta\left(y-x_{z}\right) \\
& (\partial / \partial z) K(y, z)=\rho^{2} f\left(x_{z}\right) \delta\left(y-x_{z}\right)-\rho^{2} I\left(x_{z}<y\right) \dot{f}\left(x_{z}\right) .
\end{aligned}
$$

For special cases, it is possible to solve (3.14) or (3.16) explicitly. 
Example 3.1 Suppose that $F(y)=a e^{a y}$ on $(-\infty, 0]$ where $a>0$, and that $\nu_{j}<0, \rho<-1, y \leq x$. Taking $r_{j}(0)=1$, a solution of (3.14) is

$$
r_{j}(y)=e^{b_{j} y} \text { where } b_{j}\left|\nu_{j}\right| / a=e^{b_{j} x / \rho}, b_{j}>0 .
$$

\section{Formal expressions for the eigenfunctions.}

We now give a formal solution of (3.15) for $r_{j}$ in terms of $r_{j}(0)$. (The value 0 is arbitrary: a similar solution can be obtained in terms of $r_{j}\left(y_{0}\right)$ for any $y_{0}$.) Set

$$
r(y)=r_{j}(y), c=\lambda_{j} \operatorname{sign}(\rho) .
$$

Suppose that $f$ and $r$ have Taylor series expansions about 0 . Denote the $i$ th derivatives of $f(y)$ by $f_{. i}(y)$ and set $f_{i}=f_{. i}(0), r_{i}=r_{. i}(0)$. Expanding

$$
\dot{r}(y)=c f(y) r((x-y) / \rho)
$$

about 0 , for $i \geq 0$ the coefficient of $y^{i} / i$ ! is

$$
r_{i+1}=c \sum_{a+b=i}\left(\begin{array}{l}
i \\
a
\end{array}\right) f_{a} r_{. b}(x / \rho)(-\rho)^{-b}=c \sum_{k=0}^{\infty} q_{i k} r_{k}=c f_{i} r_{0}+c \sum_{k=1}^{\infty} q_{i k} r_{k}
$$

where

$$
q_{i k}=\rho^{-k} \sum_{b=0}^{\min (i, k)}\left(\begin{array}{l}
i \\
b
\end{array}\right) f_{i-b}(-1)^{b} x^{k-b} /(k-b) ! .
$$

For $l, k \geq 1$ set $Q_{l k}=q_{l-1, k}$. Set $Q=\left(Q_{l k}: l, k \geq 1\right)$. Set

$$
\mathbf{f}^{\prime}=\left(f_{0}, f_{1}, \cdots\right), \mathbf{R}^{\prime}=\left(r_{1}, r_{2}, \cdots\right), \mathbf{Y}_{y}^{\prime}=\mathbf{Y}^{\prime}=\left(y / 1 !, y^{2} / 2 !, \cdots\right) .
$$

So $\mathbf{R}=\mathbf{f} c r_{0}+c Q \mathbf{R}, \mathbf{R}=(I-c Q)^{-1} F c r_{0}$. But $r(y)-r(0)=\mathbf{Y}^{\prime} \mathbf{R}$. So we obtain the $j$ th right eigenfunction in terms of its value at 0 :

$$
r(y) / r(0)=1+\mathbf{Y}^{\prime}\left(c^{-1} I-Q\right)^{-1} \mathbf{f},
$$

that is,

$$
r_{j}(y) / r_{j}(0)=1+\mathbf{Y}^{\prime}\left(d_{j} I-Q\right)^{-1} \mathbf{f} \text { where } d_{j}=\nu_{j} \operatorname{sign}(\rho) .
$$

For example for the extreme value distribution $F(x)=e^{-e^{-x}}, \mathbf{f}=e^{-1}(1,0,-1,-1,-7 / 288,-31 / 4, \cdots)^{\prime}$.

Since $r_{j}$ is unique only up to a constant multiplier, we may take $r_{j}(0) \equiv 1$. The solution (3.22) can now be implemented by successive approximations. For $N \geq 1$ set

$$
r_{N j}(y) / r_{j}(0)=1+\mathbf{Y}_{N}^{\prime}\left(d_{j} I_{N}-Q_{N}\right)^{-1} \mathbf{f}_{N}
$$

where $\mathbf{Y}_{N}, \mathbf{f}_{N}$ are the 1 st $N$ elements of $\mathbf{Y}, \mathbf{f}$ and $Q_{N}$ is the upper left $N \times N$ elements of $Q$. Then one expects that $r_{N j}(y) \rightarrow r_{j}(y)$ as $N \rightarrow \infty$, giving the $j$ th left eigenfunction.

A similar treatment of (3.18) gives an equation for the $j$ th left eigenfunction in terms of its value at at an arbitrary point, taken here as $x$. Set

$$
c=\rho^{2} \lambda_{j}, l=l_{j}, e(y)=f(y)^{-1} .
$$


By Taylor expansions,

$$
l(z) e(x-\rho z)=\sum_{i=0}^{\infty}\left(z^{i} / i !\right) \sum_{a+b=i}\left(\begin{array}{l}
i \\
a
\end{array}\right) l_{. a}(0) e_{. b}(x)(-\rho)^{b} .
$$

By (3.18),$l$ satisfies $(d / d z)$ LHS $=c l(x-\rho z)$. Taking the coefficient of $z^{i} / i$ !, for $i \geq 0$,

$$
\sum_{a+b=i+1}\left(\begin{array}{c}
i+1 \\
a
\end{array}\right) l_{. a}(0) e_{. b}(x)(-\rho)^{b}=c l_{. i}(x)(-\rho)^{i} .
$$

By another Taylor expansion,

$$
l_{. a}(0)=\sum_{k=0}^{\infty} l_{. k+a}(x)(-x)^{k} / k !
$$

So LHS of (3.24) is $\sum_{j=0}^{\infty} W_{i j} l_{. j}(x)=V_{i} l(x)+(W \mathbf{L})_{i}$ where we set

$$
\begin{aligned}
W_{i j} & =\sum_{a=0}^{\min (j, i+1)}\left(\begin{array}{c}
i+1 \\
a
\end{array}\right) e_{. i+1-a}(x)(-\rho)^{i+1-a}(-x)^{j-a} /(j-a) !, \\
W & =\left(W_{i j}: i, j \geq 1\right), U_{i}=W_{i 0}=e_{. i+1}(x)(-\rho)^{i+1}, V_{j}=W_{0 j}, \\
L_{j} & =l_{. j}(x), \mathbf{L}^{\prime}=\left(L_{1}, L_{2}, \cdots\right), D_{r}=\operatorname{diag}\left(r^{i}: i \geq 1\right), r=-\rho .
\end{aligned}
$$

So (3.24) for $i \geq 1$ can be written $\mathbf{U} l(x)+W \mathbf{L}=c D_{r} \mathbf{L}$ so that $\mathbf{L}=\left(c D_{r}-W\right)^{-1} \mathbf{U} l(x)$ giving in the notation of (3.21),

$$
l(z)-l(x)=\mathbf{Y}_{z-x}^{\prime} \mathbf{L}=\mathbf{Y}_{z-x}^{\prime}\left(c D_{r}-W\right)^{-1} \mathbf{U} l(x)
$$

That is, $l_{j}(z)=l(z)$ is given by

$$
l(z) / l(x)=1+\mathbf{Y}_{z-x}^{\prime}\left(c D_{r}-W\right)^{-1} \mathbf{U} .
$$

Finally, the value of the multiplier $l_{j}(x)=l(x)$ is determined by (A.3):

$$
1 / l_{j}(x)=\int r_{j}(z) R H S(\underline{3.26}) d z .
$$

\section{An equation for the eigenvalues.}

Substituting into (3.24) at $i=0$, that is $W_{00} l(x)+\mathbf{V}^{\prime} \mathbf{L}=c l(x)$, we obtain

$$
\mathbf{V}^{\prime}\left(c D_{r}-W\right)^{-1} \mathbf{U}=c-W_{00}
$$

The roots $c$ of this equation are just $\left\{\rho^{2} \lambda_{j}\right\}$, so this is the equation for the eigenvalues we have been seeking. If $\left\{\theta_{j}, j \geq 1\right\}$ are the singular values of $D_{r}^{-1} W$, and if this has diagonal Jordan form $D_{r}^{-1} W=R_{0} \Lambda L_{0}^{*}$ where $\Lambda=\operatorname{diag}\left(\theta_{1}, \theta_{2}, \cdots\right)$, (see (A.1) below), then (3.27) can be written

$$
\sum_{i=1}^{\infty} w_{i}\left(c-\theta_{i}\right)^{-1}=c-\theta_{0}
$$

where now the weights $\left\{w_{i}\right\}$ are given by

$$
w_{i}=v_{i} u_{i} \text { where } \mathbf{v}=\bar{R}_{0} D_{r}^{-1} \mathbf{V}, \mathbf{u}=L_{0}^{*} \mathbf{U} .
$$


If $\left\{c_{N j: j=1, \cdots, N+1}\right\}$ are the roots of its $N$ dimensional approximation, say

$$
\mathbf{V}_{N}^{\prime}\left(c D_{\rho N}-W_{N}\right)^{-1} \mathbf{U}_{N}=c-W_{00},
$$

then $c_{N j} \rightarrow c_{j}=\rho^{2} \lambda_{j}$ as $N \rightarrow \infty$. (This is essentially a polynomial in $c$ of degree $N+1$.) Having obtained an eigenvalue, one can substitute it into (3.22) and (3.26) to obtain the corresponding eigenfunctions up to constants $l(x)$ and $r(0)$. As noted in the appendix, either of these (but not both) can be arbitrarily chosen. The conditions $r(-\infty)=0$ and (3.17) can be verified numerically.

Example 3.2 Suppose that $f=\phi$, the density of a standard normal $\mathcal{N}(0,1)$ r.v.. Then $e_{. j}(x)=$ $\phi(x)^{-1} H_{j}^{*}(x)$ where

$$
H_{j}^{*}(x)=E(x+\mathcal{N}(0,1))^{j}=\sum_{k}\left(\begin{array}{c}
j \\
2 k
\end{array}\right) x^{j-2 k} m_{2 k}
$$

is the modified Hermite polynomial and $m_{2 k}=(2 k) ! / k ! 2^{k}$ is the $2 k$ th moment of $\mathcal{N}(0,1)$. See Withers and McGavin (2006).

An alternative is to expand $\operatorname{RHS}(\sqrt{3.24})$ about $x=0$, giving $c(-\rho)^{i}\left(l(0)+\mathbf{U}^{\prime} \mathbf{L}\right)$ where we set

$$
\begin{aligned}
L_{j} & =l_{. j}(0), \mathbf{L}^{\prime}=\left(L_{1}, L_{2}, \cdots\right), x_{k}=x^{k} / k !, \mathbf{U}^{\prime}=\left(x_{1}, x_{2}, \cdots\right), \\
A_{i a} & =l_{. a}(0)\left[e_{. b}(x)(-\rho)^{b}\right]_{b=i+1-a}, A=\left(A_{i a}: i, a \geq 1\right), V_{i}=A_{i 0}=\left[e_{. b}(x)(-\rho)^{b}\right]_{b=i+1} .
\end{aligned}
$$

Let $0^{i}$ denote the row $i$-vector of zeros. For $i \geq 1$, (3.24) gives

$$
V_{i} l(0)+\left(A_{i 1}, \cdots, A_{i, i+1}, 0,0, \cdots\right) \mathbf{L}=c(-\rho)^{i}\left(0^{i-1}, x_{0}, x_{1}, \cdots\right) \mathbf{L} .
$$

That is

$$
\mathbf{V} l(0)+A \mathbf{L}=c D_{r} X \mathbf{L}
$$

where the $i$ th row of the matrix $X$ is $\left(0^{i-1}, x_{0}, x_{1}, \cdots\right)$. So

$$
\mathbf{L}=B^{-1} \mathbf{V} l(0) \text { where } B=c D_{r} X-A, l(y) / l(0)=1+\mathbf{Y}_{y}^{\prime} B^{-1} \mathbf{V} .
$$

$X$ is upper triangular, while $A$ is lower triangular except for the 1st super-diagonal. For $i=0$, (3.24) gives

$$
\sum_{a=0}^{1} A_{0 a} l_{a}=c \sum_{k=0}^{\infty} l_{k} x_{k}=c l(0)+c \mathbf{U}^{\prime} \mathbf{L}
$$

So we obtain as an alternative equation for the eigenvalues

$$
A_{00}+A_{01}\left(B^{-1} \mathbf{V}\right)_{1}=c+c \mathbf{U}^{\prime} B^{-1} \mathbf{V} \text { where } A_{00}=-\rho e .1(x), A_{01}=e(x) .
$$

Unfortunately Appendix A cannot be applied with $\mu=F$ since $\mathcal{K} G(y)=\operatorname{sign}(\rho) \int{ }^{y} G((x-w) / \rho) d F(w)$ is not of the form $\int K(y, z) G(z) d F(z)$. It would be of great interest, and in particular allow a unified approach to this problem, if Fredholm's theory can be extended to the system

$$
\mathcal{K} \mathcal{O} r=\nu r, \mathcal{K}^{*} \mathcal{O}^{*} l=\bar{\nu} l, l_{i}^{*} \mathcal{O} r_{j} d \mu=\delta_{i j}
$$

for $\mathcal{K}$ an $q \times q$ integral operator with kernel $K(y, z): R^{p} \times R^{p} \rightarrow C^{q \times q}$ with respect a measure $\mu, \mathcal{O}$ a $q \times q$ operator, where $*$ is the transpose of the complex conjugate, and $\bar{\nu}$ is the complex conjugate of $\nu$.

For our problem, one could then apply the theory with

$$
p=q=1, \mu=F, K(y, z)=\operatorname{sign}(\rho) I(z<y), \mathcal{O} G(w)=G((x-w) / \rho) .
$$




\section{APPENDIX A}

To make the paper self-contained, we give here some theory for Fredholm integral equations with non-symmetric kernels.

First consider the case where $K$ is any $k \times k$ complex matrix. Its singular value decomposition is

$$
K=R \Lambda L^{*} \text { where } R R^{*}=I, L L^{*}=I, \Lambda=\operatorname{diag}\left(\nu_{1}, \nu_{2}, \cdots\right),
$$

* denotes the complex conjugate transpose. Since

$$
K K^{*} R=R \Lambda \Lambda^{*}, K^{*} K L=L \Lambda^{*} \Lambda,
$$

the $j$ th column of $R$ is a right eigenvector of $K K^{*}$ with eigenvalue $\left|\lambda_{j}\right|^{2}$ and the $j$ th column of $L$ is a right eigenvector of $K^{*} K$ with the same eigenvalue. If $K$ is non-singular, its inverse is

$$
K^{-1}=L \Lambda^{-1} R^{*} .
$$

If it is singular, a pseudoinverse is given by

$$
K^{-}=L \Lambda^{-} R^{*} .
$$

However the singular value decomposition does not give a nice form for powers of $K$. This drawback is overcome by its Jordan decomposition. Consider the case where this is diagonal. Then

$$
K=R \Lambda L^{*} \text { where } R L^{*}=I, \text { and } \Lambda=\operatorname{diag}\left(\nu_{1}, \nu_{2}, \cdots,\right)
$$

is composed of the eigenvalues of $K$. Then for any complex $\alpha$,

$$
K^{\alpha}=R \Lambda^{\alpha} L^{*}
$$

Taking $\alpha=n$ and $\alpha=-1$ gives the $n$th power and inverse of $K$.

Now let $K(y, z)$ be a real function on $\Omega \times \Omega$ where $\Omega$ is a subset of $R^{p}$. Suppose that $\mu$ is a $\sigma$-finite measure on $\Omega$ and that

$$
0<\|\mathcal{K}\|_{2}^{2}=\iint K(y, z) K(z, y) d \mu(y) d \mu(z)<\infty .
$$

(This $L_{2}$ condition can be changed to

$$
\int|K(y, y)| d \mu(y)<\infty
$$

at the expense of notational complexities that need not concern us here.) The corresponding integral operator $\mathcal{K}$ is defined by

$$
\mathcal{K} \phi(y)=\int K(y, z) \phi(z) d \mu(z), \phi(z) \mathcal{K}=\int \phi(y) K(y, z) d \mu(y) .
$$

The Fredholm equations of the first kind,

$$
\lambda \mathcal{K} r(y)=r(y), \lambda l(z) \mathcal{K}=l(z),
$$


have only a countable number of solutions, say $\left\{\lambda_{j}, r_{j}(y), l_{j}(z), j \geq 1\right\}$ up to arbitrary constant multipliers for $\left\{r_{j}(y), j \geq 1\right\}$, and these satisfy

$$
\int r_{j} l_{k} d \mu=\delta_{j k} \text { where } \int g d \mu=\int_{R^{p}} g(y) d \mu(y) .
$$

These are called the singular values (or eigenvalues) and right and left eigenfunctions of $(K, \mu)$ or $\mathcal{K}$. Also

$$
K(y, z)=\sum_{j=1}^{\infty} r_{j}(y) l_{j}(z) / \lambda_{j}
$$

with convergence in $L_{2}(\mu \times \mu)$, or more strongly under other conditions: see Withers $(1974,1975$, 1978). This is the functional form of the Singular Value Decomposition for a square non-symmetric matrix.

Fredholm equations of the second kind,

$$
r(y)-\lambda \mathcal{K} r(y)=f(y), l(z)-\lambda l(z) \mathcal{K}=g(z),
$$

can be solved for $\lambda$ not an eigenvalue using

$$
(I-\lambda \mathcal{K})^{-1}=I+\lambda \mathcal{K}_{\lambda}
$$

where

$$
\mathcal{K}_{\lambda} f(y)=\int K(y, z, \lambda) f(z) d \mu(z), g(z) \mathcal{K}_{\lambda}=\int g(y) K(y, z, \lambda) d \mu(y)
$$

and the resolvent $K(y, z, \lambda)$ with operator $\mathcal{K}_{\lambda}$ is the unique solution of

$$
(I-\lambda \mathcal{K}) \mathcal{K}_{\lambda}=\mathcal{K}=\mathcal{K}_{\lambda}(I-\lambda \mathcal{K}),
$$

that is,

$$
\lambda \int K(y, u) K(u, z, \lambda) d \mu(u)=K(y, z)-K(y, z, \lambda)=\lambda \int K(y, u, \lambda) K(u, z) d \mu(u) .
$$

If this can be solved analytically or numerically, then often one does not need to compute the eigenvalues and eigenfunctions. Alternatively, the resolvent satisfies

$$
K(y, z, \lambda)=\sum_{j=1}^{\infty} r_{j}(y) l_{j}(z) /\left(\lambda_{j}-\lambda\right) .
$$

The Fredholm determinant is

$$
D(\lambda)=\Pi_{j=1}^{\infty}\left(1-\lambda / \lambda_{j}\right)=\exp \left\{-\int_{0}^{\lambda} d \lambda \int K(y, y, \lambda) d \mu(y)\right\} .
$$

Note that

$$
\|\mathcal{K}\|_{2}^{2}=\sum_{j=1}^{\infty} \lambda_{j}^{-2}
$$


Also since $\left(1-\lambda / \lambda_{j}\right)^{-1}-1=\lambda /\left(\lambda_{j}-\lambda\right)$,

$$
\lambda K(y, z, \lambda)=\sum_{j=1}^{\infty} r_{j}(y) l_{j}(z)\left[\left(1-\lambda / \lambda_{j}\right)^{-1}-1\right] .
$$

If only a finite number of eigenvalues are non-zero, the kernel $K(y, z)$ is said to be degenerate. (For example this holds if $\mu$ puts weight only at $n$ points.) If not, $\left\{l_{j}\right\}$ and $\left\{r_{j}\right\}$ typically both span $L_{2}(\mu)=\left\{f: \int|f|^{2} d \mu<\infty\right\}$. For $f \in L_{2}(\mu)$,

$$
f(y)=\sum_{j=1}^{\infty} R_{j} r_{j}(y)=\sum_{j=1}^{\infty} L_{j} l_{j}(y) \text { where } R_{j}=\int f l_{j} d \mu, L_{j}=\int f r_{j} d \mu
$$

with convergence in $L_{2}(\mu)$. So

$$
\mathcal{K}^{n} f(y)=\sum_{j=1}^{\infty} R_{j} r_{j}(y) / \lambda_{j}^{n}, f(y) \mathcal{K}^{n}=\sum_{j=1}^{\infty} L_{j} l_{j}(y) / \lambda_{j}^{n}, n \geq 0 .
$$

So if

$$
\left|\lambda_{1}\right|<\left|\lambda_{j}\right|
$$

for $j>1$ then as $n \rightarrow \infty$,

$$
\mathcal{K}^{n+1} f(y) / \mathcal{K}^{n} f(y) \rightarrow \lambda_{1}^{-1}, f(y) \mathcal{K}^{n+1} / f(y) \mathcal{K}^{n} \rightarrow \lambda_{1}^{-1} .
$$

This is one way to obtain $\lambda_{1}$ arbitrarily closely. Another is to use

$$
\begin{gathered}
\lambda_{1}^{-1}=\sup \left\{\int g \mathcal{K} h d \mu: \int g h d \mu=1\right\} \text { if } \lambda_{1}>0, \\
\lambda_{1}^{-1}=\inf \left\{\int g \mathcal{K} h d \mu: \int g h d \mu=1\right\} \text { if } \lambda_{1}<0 .
\end{gathered}
$$

The maximising/minimising functions are the first eigenfunctions $g=g_{1}, h=h_{1}$. These are unique up to a constant multiplier if (A.9) holds. If $\lambda_{1}$ is known, one can use

$$
\left(\lambda_{1} \mathcal{K}\right)^{n} f(y) \rightarrow R_{1} r_{1}(y), f(y)\left(\lambda_{1} \mathcal{K}\right)^{n} \rightarrow L_{1} l_{1}(y),
$$

to approximate $R_{1} r_{1}(y), L_{1} l_{1}(y)$. Also since $l_{1}(y)$ is only unique up to a multiplicative constant, we may choose $R_{1}=1$ and so approximate $r_{1}(y), l_{1}(y)$. One may now repeat the procedure on the operator $\mathcal{K}_{1}$ corresponding to

$$
K_{1}(y, z)=K(y, z)-r_{1}(y) l_{1}(z)
$$

to approximate $\lambda_{2}, r_{2}(y), l_{2}(z)$, assuming the next eigenvalue in magnitude, $\lambda_{2}$, has multiplicity 1 . If say $\lambda_{1}$ has multiplicity $M>1$, then

$$
\left(\lambda_{1} \mathcal{K}\right)^{n} f(y) \rightarrow \sum_{j=1}^{M} R_{j} r_{j}(y)
$$

and one can adapt the method above.

For further details see Withers and Nadarajah (In press). For further details on Fredholm theory for symmetric kernels, see Withers $(1974,1975,1978)$.

\section{References}

[1] Comtet, L. (1974) Advanced combinatorics. Reidel, Dordrecht. 
[2] Leadbetter M. R., Lindgren G., and Rootzen H. (1983) Extremes and related properties of random sequences, Springer-Verlag, New York.

[3] Pogorzelski, W. (1966) Integral equations and their applications, Volume 1, Pergamon, Oxford.

[4] Resnick, S. I. (1987) Extreme values, regular variation, and point processes. Springer-Verlag, New York.

[5] Stuart, A. and Ord, K. (1987). Kendall's advanced theory of statistics, 1. 5th edition. Griffin, London.

[6] Withers, C. S. (1974) Mercer's Theorem and Fredholm resolvents. Bull. Austral. Math. Soc., 11, 373-380.

[7] Withers, C. S. (1975) Fredholm theory for arbitrary measure spaces. Bull. Austral. Math. Soc., No. 2, 283-292.

[8] Withers, C. S. (1978) 1978 Fredholm equations have uniformly convergent solutions. Jnl. of Math. Anal. and Applic., 64, 602-609.

[9] Withers, C. S. and McGavin, P. (2006) Expressions for the normal distribution and repeated normal integrals, Statistics and Probability Letters, 76, No. 5, 479-487.

[10] Withers, C. S. and Nadarajah, S. (In press) Fredholm equations for non-symmetric kernels with applications to iterated integral operators, Applied Mathematics and Computation. 